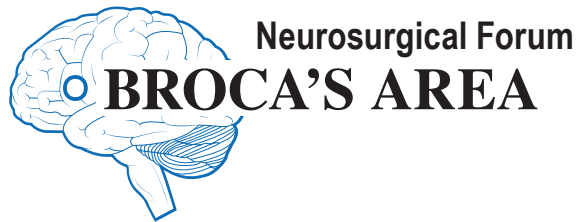

\section{A call for consistent radiographic definition of lumbar lordosis}

\author{
Mark B. Frenkel, MD, ${ }^{1}$ Casey D. Frey, BS, ${ }^{2}$ \\ Jaclyn J. Renfrow, MD, ${ }^{1}$ Stacey Q. Wolfe, MD, ${ }^{1}$ \\ Alexander K. Powers, MD, ${ }^{1}$ and Charles L. Branch Jr., MD'
}

1 Department of Neurosurgery, Wake Forest Baptist Medical Center, Winston-Salem, North Carolina

${ }^{2}$ Wake Forest University School of Medicine, Winston-Salem,

North Carolina

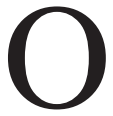

VER the past several years there has been increased interest in lumbar lordosis (LL) and its relationship to pathology and other spinopelvic parameters, especially pelvic incidence (PI). Lumbar lordosis has been shown to correlate strongly enough with clinical outcome measures in patients with adult spinal deformity (ASD) that some authors suggest its restoration should be a primary objective of deformity surgery. ${ }^{18}$ Unfortunately, the measurement of LL with other spinopelvic parameters has become such a norm that some studies ${ }^{11}$ neglect to even mention it in their Methods section. We have identified multiple variations in the definition of LL throughout the literature, and hypothesize that these variations may impact the conclusions and clinical decision making that derives from this measurement. In this paper we attempt to determine how pervasive the variability of the definition of LL is within the current spine literature.

\section{Methods}

\section{Search Strategy}

A literature search of PubMed was conducted by searching for the term "Lumbar Lordosis" in the Title, Keyword, and/or Abstract fields. The search was restricted to papers published between January 1, 2016, and December 31, 2016. Publications that originally appeared in the query results as "published ahead of print" but that were subsequently updated with later publication dates were not removed. The search was also restricted to the following spine-related journals: Spine; Clinical Spine Surgery; The
Spine Journal; Journal of Neurosurgery: Spine; Neurosurgery; Asian Spine Journal; International Journal of Spine Surgery; Spine Deformity; Scoliosis; Scoliosis and Spinal Disorders; and Global Spine Journal. Papers were screened by 2 authors and included only if they measured LL in at least 1 patient for the purposes of the study.

\section{Data Extraction}

The entirety of each paper was reviewed twice (once each by 2 authors) using a standardized form. The authors recorded whether each study explicitly specified the upper and lower vertebrae from which LL was measured and, if so, whether the study specified if the inferior or superior endplates of each vertebra was used (with the exception of those measurements terminating at $\mathrm{S}-1$, in which case it was only recorded whether the endplate of the rostral vertebra was specified). Discrepancies between reviewers prompted a reevaluation of the article in question by both reviewers.

\section{Results}

Of the 118 papers initially identified, 5 (2 editorials, 2 review articles, and 1 research study) were excluded for not measuring LL in at least 1 patient. A total of 113 papers were included and critically reviewed. Thirty-four studies $(30 \%)$ failed to describe which vertebrae were used to measure LL. One mischaracterized segmental lordosis as LL. One used a nonconstrained measurement. Three measured surface topography. Three studies ${ }^{1,6,8}$ had diagrams illustrating LL but did not explicitly state the vertebrae used for measurement in the text of the manuscript or figure legend. These studies were considered to be lacking a definition, because a nonconstrained measurement could have been used. Of the 79 papers that did specify the rostral and caudal vertebrae from which LL was measured, 21 did not specify which endplate was used for the measurement, leaving only 58 studies $(51 \%)$ with complete definitions of LL.

In those papers that did define which vertebrae were used to measure LL, 12 different definitions of lumbar lordosis were used (Table 1). This lack of consistency 
held true even when comparing papers published within individual journals; only the 4 papers published in Neurosurgery had a consistent definition within the same journal (although this could not be assessed for International Journal of Spine Surgery or Global Spine Journal because these each had only 1 paper that met inclusion criteria).

The included papers used the measure of LL for several different purposes. Thirty-nine $(34.5 \%)$ of the included papers used LL to assess the impact of an intervention or compare interventions (Supplemental Table 1). Twentyone (18.6\%) used LL to assess the reliability of spinopelvic measures or the relationship between spinopelvic and other physiological or quality of life measures (Supplemental Table 2). Thirty-two (28.3\%) used LL measurements to assess whether some values of LL were risk factors for disease, predictors of a complication or outcome, or correlated with disease severity (Supplemental Table 3). Twenty-one (18.6\%) used LL measurements to define a disease state or normal parameters in a population (Supplemental Table 4). There was no consistent definition of LL used within any of these groups. These studies also examined several different cohorts: 10 were focused on healthy individuals, 32 on nonoperatively treated or preoperative patients with disease, 70 on postoperative patients, and 1 study was performed on cadavers. Of the 10 studies focused on healthy individuals, 9 of them described how LL was measured, and 5 of these used the same definition (one used the superior endplate of L1-S1; one did not specify which endplates of L1-S1 were used; another used a nonconstrained measurement; and two used surface topography). All of these 10 were used to either assess the reliability of or relationship between spinopelvic measures, or to define normal parameters in a population.

Twenty-one papers focused primarily on some form of scoliosis, with 7 focusing on either adolescent idiopathic or congenital scoliosis. There was no consistency in the measure of LL used within any of these groups.

\section{Discussion}

\section{Impact of Different Definitions}

The lack of a consistent definition of LL was first brought to light by Polly et al. more than 20 years ago when they identified 4 different definitions of LL in the popular literature and examined the interobserver reliability of each. ${ }^{15}$ We found 12 different definitions of LL used among the studies we analyzed. The most obvious implication of these differences is that uncertainty is introduced when making comparisons between studies. For illustration, the T-12 and L-1 vertebral bodies have each been shown to contribute an average of approximately $4^{\circ}$ of kyphosis due to anterior wedging. ${ }^{7}$ This has been corroborated by other studies, with at least one population demonstrating statistically significant differences in vertebral body angulation between sexes. ${ }^{2,14}$ Similarly, the L5-S1 disc space has been shown to contribute an average of $11.7^{\circ}$ of lordosis in at least one population, ${ }^{3}$ and the segmental angle between the superior endplate of L-5 and the superior endplate of S-1 (i.e., both the L-5 vertebral body and L5-S1 disc space combined) has been shown to contribute an average of approximately $25^{\circ}$ of lordo-
TABLE 1. The number of papers in the 2016 literature using each definition of $L L$

\begin{tabular}{lc}
\hline \multicolumn{1}{c}{ Definition of LL } & No. of Papers \\
\hline Superior L1-superior L5 & 1 \\
\hline Superior L1-inferior L5 & 5 \\
\hline Endplates unknown, L1-L5 & 2 \\
\hline Superior L1-superior S1 & 33 \\
\hline Inferior L1-superior S1 & 1 \\
\hline Endplates unknown, L1-S1 & 12 \\
\hline Inferior T12-superior S1 & 9 \\
\hline Superior T12-superior S1 & 9 \\
\hline Endplates unknown, T12-S1 & 7 \\
\hline Nonconstrained Cobb angle & 1 \\
\hline Surface topography & 3 \\
\hline Segmental lordosis & 1 \\
\hline Not stated & 29 \\
\hline
\end{tabular}

sis, ${ }^{12}$ suggesting an approximately $13^{\circ}$ contribution from the L-5 vertebral body. As a result, the difference between measuring from the superior endplate of T- 12 to S-1 versus the superior endplate of L-1 to the inferior endplate of L-5 (as was done in 9 and 5 of the studies reviewed, respectively) can easily exceed the $11^{\circ}$ PI-LL mismatch commonly accepted to be clinically significant for $\mathrm{LL}_{\mathrm{sL} 1-\mathrm{S} 1} \cdot{ }^{19} \mathrm{In}$ fact, one group found an $18.3^{\circ}$ difference between the means of 90 measurements each of $\mathrm{LL}_{\mathrm{iT12}-\mathrm{S} 1}$ and $\mathrm{LL}_{\mathrm{sL} 1-\mathrm{iL} 5}$ on 30 radiographs. ${ }^{10}$

These discrepancies in the definition of LL create an inconsistent platform for the use of PI-LL as a clinical tool. This was demonstrated by one study in which logistic regression was used to determine a cutoff for a PI-LL value that had adequate sensitivity and reliability for predicting the risk of developing adjacent-segment disease in patients with prior lumbar fusions. Calculations were performed for both $\mathrm{LL}_{\mathrm{sL} 1-\mathrm{S} 1}$ and $\mathrm{LL}_{\text {?L1-2L5}}$, and the authors found a 55\% higher cutoff value for the latter $\left(9.8^{\circ}\right.$ and $15.2^{\circ}$, respectively), further illustrating the impact of different LL definitions on PI-LL and the clinical decisions made from this calculation. ${ }^{16}$

Unfortunately, the lack of a consistent definition of LL is pervasive throughout the literature. In some cases, the same primary author or large spine study group have used different definitions in different publications. ${ }^{20,21}$ As a result of both how common the use of different LL definitions is and the potential impact of different definitions on a study's findings, questions are raised by those studies that fail to specify the definition of LL used. Take for example a large multicenter trial in which it was concluded that there was no correlation between LL and patient satisfaction scores in postoperative patients with ASD. The authors did not specify the definition of LL they used for measurement, raising the question of whether uniform measurements were used between study sites. ${ }^{9}$

\section{A Call for Consensus}

We have found that the literature is rife with studies whose methodology or conclusion has been negatively 


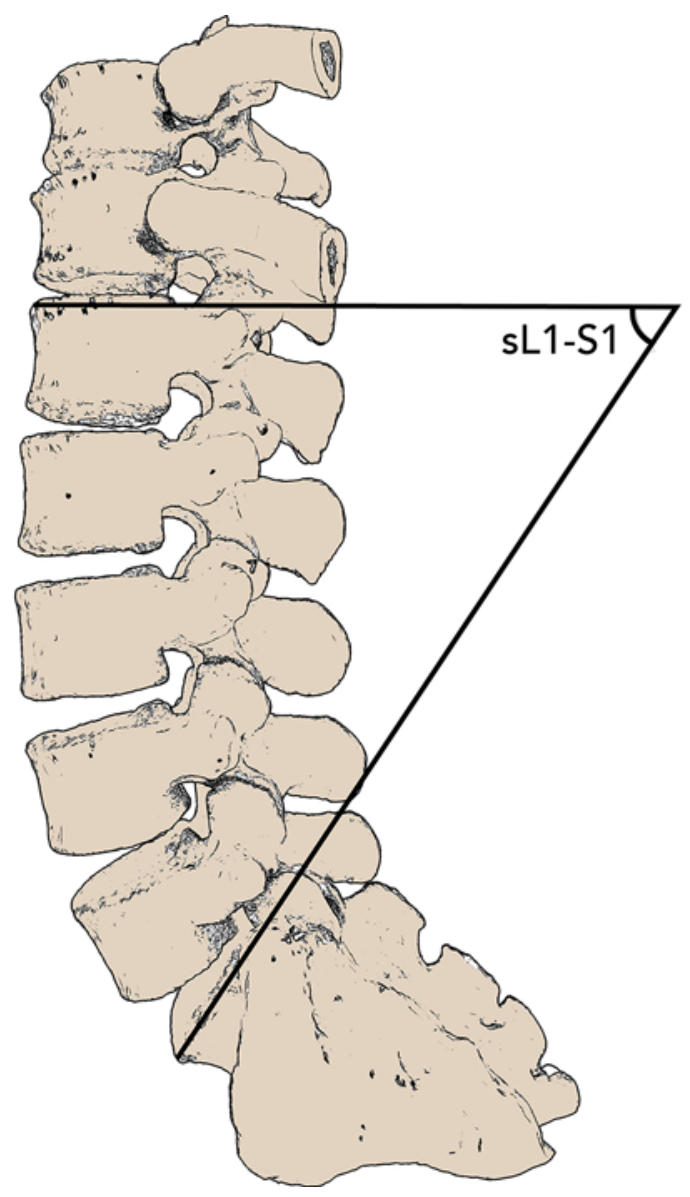

FIG. 1. Illustration showing our proposed standardized definition of $L L$ as measured from the superior endplate of $\mathrm{L}-1$ to the superior endplate of S-1. Copyright Mark Frenkel. Published with permission. Figure is available in color online only.

impacted by lack of a strict universal definition of LL. We propose that the angle formed by the intersection of a line tangent to the superior endplate of L-1 with a second line tangent to the superior endplate of S-1 be used as the common measure of LL (Fig. 1). The S-1 level was chosen rather than L-5 because of the large degree of lordosis provided by the L5-S1 disc. The L-1 level was chosen rather than T-12 because the transitional vertebrae between thoracic kyphosis and LL are at or below L-1 in the majority of patients. ${ }^{22}$ In addition, this was the most commonly used definition in the literature we examined from 2016.

Although the issues arising from the use of different definitions of LL can be avoided in the future by using the single standardized definition proposed above, this may be unrealistic because it would require extensive collaboration. In addition, a new dimension of difficulty is introduced when referencing papers that have already been published. This difficulty can be seen in a 2013 paper, ${ }^{18}$ which provides a table with the "LL (L1-S1)" from 6 prior papers. However, of these papers, one ${ }^{4}$ measured a nonconstrained LL to iL5, two $^{5,22}$ measured a nonconstrained LL to $\mathrm{S}-1$, another ${ }^{13}$ measured $\mathrm{LL}_{\text {?T12-?L5 }}$, and the final one measured "S1 lordosis," which they failed to describe. ${ }^{17}$

We propose that the best method for avoiding uncer-
TABLE 2. Proposed shortened nomenclature for definitions of LL

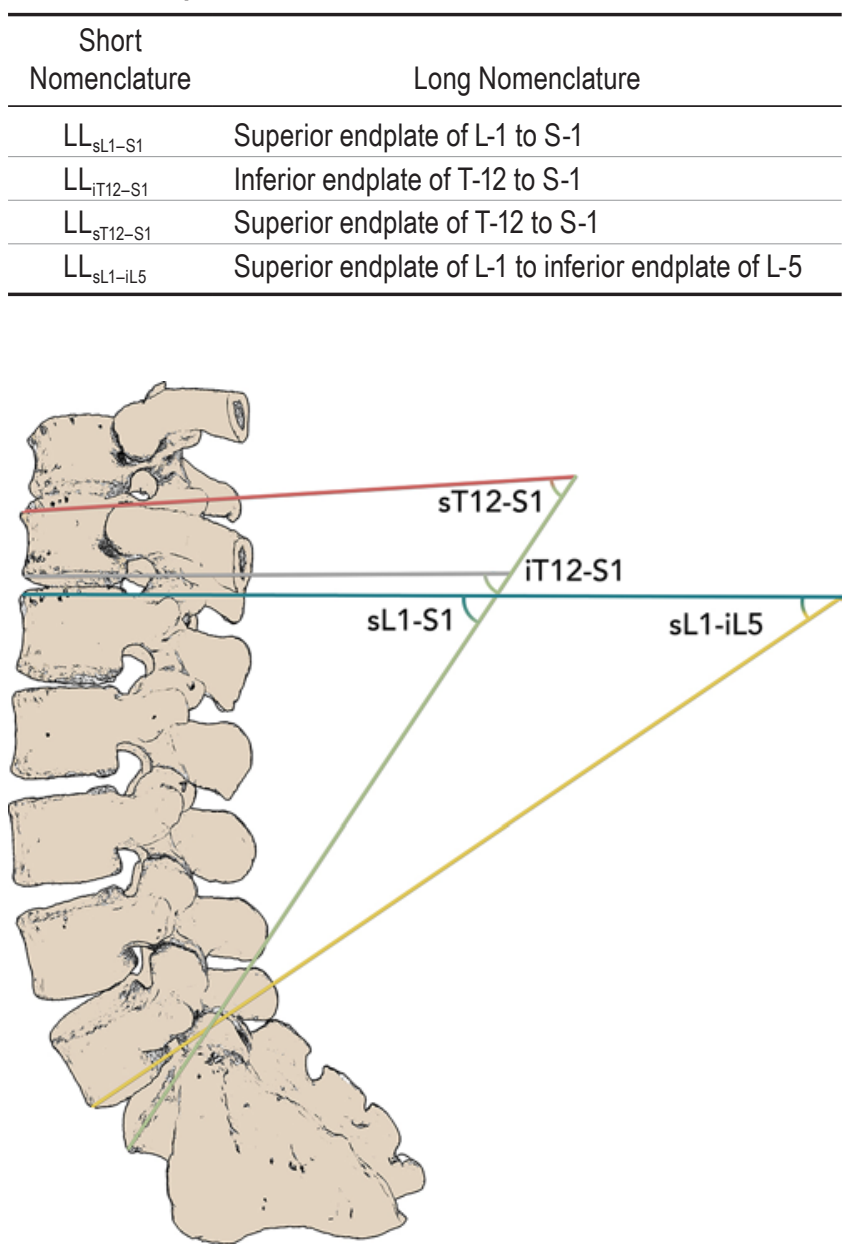

FIG. 2. Illustration showing the 4 most common definitions of $L L$ that appeared in the 2016 literature, along with our new descriptive nomenclature. Copyright Mark Frenkel. Published with permission. Figure is available in color online only.

tainty in the future is with the introduction of a new nomenclature for LL (Table 2). Ideally, this should replace every instance where the term "LL" appears in a manuscript. It is as follows.

1) LL should be followed by a subscript indicating the levels from which it was measured.

2) The most rostral vertebra measured should be named first, followed by a dash, and then the most caudal vertebra.

3) Levels in the thoracic spine, such as T-12, should include the "T" prefix. The same applies to " $\mathrm{S}$ " for levels in the sacrum and " $L$ " for lumbar levels.

4) The endplate used for measurement should be identified with either a lowercase " $i$ " for the inferior endplate or a lowercase "s" for the superior endplate preceding the level. A "?" should be used if the endplate used is unknown.

5) The endplate identifier is not necessary for S-1 because it is assumed that the superior endplate is used.

6) Nonstandard or calculated values of LL may be de- 
noted with an " $x$ " in subscript to refer the user to the Methods section of the manuscript.

For example, LL measured from the superior endplate of L-1 to the inferior endplate of L-5 would be described as $\mathrm{LL}_{\mathrm{sL} 1-\mathrm{iL} 5}$ (Fig. 2). Likewise, a measurement from the superior endplate of T-12 to S-1 would be $\mathrm{LL}_{\mathrm{ST} 12-\mathrm{S} 1}$. The use of a subscript allows this nomenclature to be used in situations such as the PI-LL equation without having a significant impact on formatting.

\section{Conclusions}

Our analysis of the 2016 spine literature has revealed a high degree of variability in the definitions of LL used in different manuscripts. This lack of consensus introduces a degree of uncertainty in comparing the findings of different studies, and may have a significant impact on the clinical relevance of PI-LL differences. We propose that a standardized definition of LL, from the superior endplate of L-1 to the superior endplate of S-1, be adopted and that authors and editors alike remain vigilant in its use. Furthermore, we have introduced a proposed nomenclature for when this definition cannot be used or when referencing prior studies.

\section{References}

1. Ames C, Gammal I, Matsumoto M, Hosogane N, Smith JS, Protopsaltis T, et al: Geographic and ethnic variations in radiographic disability thresholds: analysis of North American and Japanese operative adult spinal deformity populations. Neurosurgery 78:793-801, 2016

2. Ayad CE, Abdalla EA, Osman AM, Mohammed ME: Defining normal vertebral end plates Cobb angle from T12 to L4 using computerized tomography in Sudanese populations. Int J Med Imaging 1:66-70, 2014

3. Been E, Barash A, Pessah H, Peleg S: A new look at the geometry of the lumbar spine. Spine (Phila Pa 1976) 35:E1014-E1017, 2010

4. Berthonnaud E, Dimnet J, Roussouly P, Labelle H: Analysis of the sagittal balance of the spine and pelvis using shape and orientation parameters. J Spinal Disord Tech 18:40-47, 2005

5. Boulay C, Tardieu C, Hecquet J, Benaim C, Mouilleseaux B, Marty C, et al: Sagittal alignment of spine and pelvis regulated by pelvic incidence: standard values and prediction of lordosis. Eur Spine J 15:415-422, 2006

6. Chung NS, Jeon CH, Lee HD, Won SH: Measurement of spinopelvic parameters on standing lateral lumbar radiographs: validity and reliability. Clin Spine Surg 30:E119-E123, 2017

7. Crawford MB, Toms AP, Shepstone L: Defining normal vertebral angulation at the thoracolumbar junction. AJR Am J Roentgenol 193:W33-W37, 2009

8. Gupta M, Henry JK, Schwab F, Klineberg E, Smith JS, Gum $\mathrm{J}$, et al: Dedicated spine measurement software quantifies key spino-pelvic parameters more reliably than traditional picture archiving and communication systems tools. Spine (Phila Pa 1976) 41:E22-E27, 2016

9. Hamilton DK, Kong C, Hiratzka J, Contag AG, Ailon T, Line $B$, et al: Patient satisfaction after adult spinal deformity surgery does not strongly correlate with health-related quality of life scores, radiographic parameters or occurrence of complications. Spine (Phila Pa 1976) 42:764-769, 2017

10. Harrison DE, Harrison DD, Cailliet R, Janik TJ, Holland B: Radiographic analysis of lumbar lordosis: centroid, Cobb,
TRALL, and Harrison posterior tangent methods. Spine (Phila Pa 1976) 26:E235-E242, 2001

11. Hey HWD, Tan KA, Neo CSE, Lau ETC, Choong DAW, Lau LL, et al: T9 versus T10 as the upper instrumented vertebra for correction of adult deformity-rationale and recommendations. Spine J 17:615-621, 2017

12. Lee ES, Ko CW, Suh SW, Kumar S, Kang IK, Yang JH: The effect of age on sagittal plane profile of the lumbar spine according to standing, supine, and various sitting positions. J Orthop Surg Res 9:11, 2014

13. Legaye J, Duval-Beaupère G, Hecquet J, Marty C: Pelvic incidence: a fundamental pelvic parameter for three-dimensional regulation of spinal sagittal curves. Eur Spine J 7:99-103, 1998

14. Masharawi Y, Dar G, Peleg S, Steinberg N, Medlej B, May $\mathrm{H}$, et al: A morphological adaptation of the thoracic and lumbar vertebrae to lumbar hyperlordosis in young and adult females. Eur Spine J 19:768-773, 2010

15. Polly DW Jr, Kilkelly FX, McHale KA, Asplund LM, Mulligan M, Chang AS: Measurement of lumbar lordosis. Evaluation of intraobserver, interobserver, and technique variability. Spine (Phila Pa 1976) 21:1530-1536, 1996

16. Rothenfluh DA, Mueller DA, Rothenfluh E, Min K: Pelvic incidence-lumbar lordosis mismatch predisposes to adjacent segment disease after lumbar spinal fusion. Eur Spine J 24:1251-1258, 2015

17. Roussouly P, Gollogly S, Noseda O, Berthonnaud E, Dimnet $\mathrm{J}$ : The vertical projection of the sum of the ground reactive forces of a standing patient is not the same as the $\mathrm{C} 7$ plumb line: a radiographic study of the sagittal alignment of 153 asymptomatic volunteers. Spine (Phila Pa 1976) 31:E320E325, 2006

18. Schwab FJ, Blondel B, Bess S, Hostin R, Shaffrey CI, Smith JS, et al: Radiographical spinopelvic parameters and disability in the setting of adult spinal deformity: a prospective multicenter analysis. Spine (Phila Pa 1976) 38:E803-E812, 2013

19. Schwab F, Ungar B, Blondel B, Buchowski J, Coe J, Deinlein D, et al: Scoliosis Research Society-Schwab adult spinal deformity classification: a validation study. Spine (Phila Pa 1976) 37:1077-1082, 2012

20. Smith JS, Lafage V, Shaffrey CI, Schwab F, Lafage R, Hostin R, et al: Outcomes of operative and nonoperative treatment for adult spinal deformity: a prospective, multicenter, propensity-matched cohort assessment with minimum 2-year follow-up. Neurosurgery 78:851-861, 2016

21. Smith JS, Singh M, Klineberg E, Shaffrey CI, Lafage V, Schwab FJ, et al: Surgical treatment of pathological loss of lumbar lordosis (flatback) in patients with normal sagittal vertical axis achieves similar clinical improvement as surgical treatment of elevated sagittal vertical axis: clinical article. J Neurosurg Spine 21:160-170, 2014

22. Vialle R, Levassor N, Rillardon L, Templier A, Skalli W, Guigui P: Radiographic analysis of the sagittal alignment and balance of the spine in asymptomatic subjects. J Bone Joint Surg Am 87:260-267, 2005

\section{Disclosures}

Dr. Branch is a consultant for and a patent holder with Medtronic.

\section{Supplemental Information \\ Online-Only Content}

Supplemental material is available with the online version of the article.

Supplemental Tables. https://thejns.org/doi/suppl/10.3171/ 2017.11.SPINE17976. 